\title{
Múineadh Ábhar tríd an Tríú Teanga i mBunscoileanna Lán-Ghaeilge
}

\author{
Aisling Ní Dhiorbháin \\ Ollscoil Chathair bhaile Átha Cliath \\ aisling.nidhiorbhain@dcu.ie
}

\section{Achoimre}

Déantar cur síos san alt seo ar thaithí cúigear múinteoirí agus príomhoide amháin ar an bhFoghlaim Chomhtháite Ábhar agus Teangacha (FCÁT) a chur i bhfeidhm tríd an tríú teanga i mbunscoileanna lán-Ghaeilge. Tá cáil ar FCÁT mar chur chuige atá éifeachtach leis an dara teanga a theagasc. Is éard atá i gceist le FCÁT, ná go múintear ábhar nó cuid d'ábhar trí mheán an dara teanga nó teanga bhreise. $\mathrm{Ba}$ scéim phíolótach cheannródaíoch a bhí sa taighde seo mar go raibh FCÁT á chur i bhfeidhm le foghlaimeoirí óga, ag baint leasa as an tríú teanga i suíomh an tumoideachais lán-Ghaeilge. Chomhtháthaigh na múinteoirí a bhí páirteach sa taighde, teanga Eorpach (An Fhraincis/An Ghearmáinis), leis an bhfoghlaim ábhair in ábhar scoile amháin le linn na scoilbhliana. Bhí réimse rang-ghrúpaí agus ábhar i gceist agus cuireadh múnlaí éagsúla de FCÁT i bhfeidhm ag brath ar inniúlacht na múinteoirí sa sprioctheanga. Bailíodh eolas cáilíochtúil faoi eispéiris na múinteoirí ó cheistneoir agus grúpa fócais. Cé gur taighde ar scála beag atá sa taighde seo, is ábhar taighde nuálach é a thugann léargas ar chuid de na saincheisteanna a bheadh le plé dá mbeadh FCÁT le cur i bhfeidhm i scoileanna lán-Ghaeilge ar bhonn níos leithne amach anseo. Eascraíonn ceisteanna maidir leis na hábhair seo a leanas: inniúlacht múinteoirí sa sprioctheanga, forbairt ghairmiúil leanúnach do mhúinteoirí, torthaí foghlama teanga FCÁT agus soláthar áiseanna do FCÁT. Is í an phríomhcheist a eascraíonn ón taighde ná, cén múnla FCÁT ab fheiliúnaí do shainchomhthéacs na mbunscoileanna lán-Ghaeilge in Éirinn.

\footnotetext{
Abstract

This article reports the experiences five Irish-medium primary teachers, and one Irish-medium principal of implementing Content and Integrated Learning (CLIL) through a third language in Irish-medium primary schools. CLIL is recognised as an effective approach to second language teaching. It entails the teaching of a
} 
subject, or a part of a subject through the medium of a second or an additional language. In this pioneering pilot study CLIL was implemented with young learners through a third language in an Irish-medium immersion setting. The teachers involved in the project integrated a European language (French/German), with content learning in one subject throughout the school year. There were a variety of class groups and subjects involved, and teachers adopted various approaches to CLIL in accordance with their own linguistic proficiency. Data relaying the teachers' experiences and approaches to CLIL were gathered from a questionnaire and focus group interview. This small scale innovative research provides an insight into prominent questions for discussion if CLIL is to be implemented in Irishmedium schools on a wider basis. Questions arise concerning: language teacher proficiency, continuous professional development for teachers, language learning outcomes for CLIL, and the provision of specifically designed CLIL resources. A key question arising from the study is what model of CLIL would be most suitable for the specific context of Irish-medium primary schools in Ireland.

Eochairfhocail: CLIL / FCÁT, Tríú teanga, Bunscoileanna lán-Ghaeilge, Saineolas ábhar oideolaíoch, Forbairt ghairmiúil

\section{Réamhrá}

Aithnítear borradh na scoileanna lán-Ghaeilge in Éirinn mar an gníomh is dearfaí agus is éifeachtaí maidir le cur chun cinn na Gaeilge ó bunaíodh an Saorstát (Comhairle na hEorpa, 2008; Ó Ceallaigh \& Ní Dhonnabháin, 2015). De bharr éilimh, tá líon na mbunscoileanna lánGhaeilge ag méadú gach bliain, agus tuairiscíodh go raibh 245 bunscoil, is é sin nach mór 8\% de na bunscoileanna ar fad, ag múineadh trí mheán na Gaeilge sa scoilbhliain 2019 (An Roinn Oideachais \& Scileanna, 2020). Is tumchur chuige iomlán a chuirtear i bhfeidhm sna bunscoileanna lán-Ghaeilge ina múintear gach ábhar seachas an Béarla trí mheán na Gaeilge. Cosúil le scoileanna tumoideachais i ndlínsí eile (Swain \& Johnson, 1997), is é an dátheangachas suimitheach atá mar sprioc ag na bunscoileanna lán-Ghaeilge. Is é sin le rá, go n-éireoidh le foghlaimeoirí lán-Ghaeilge ardchaighdeán líofachta a bhaint amach sa Ghaeilge, gan aon bhac a chothú do ghnóthachtáil sa chéad teanga. Is é Béarla máthairtheanga formhór na bhfoghlaimeoirí lán-Ghaeilge. I gcomparáid le foghlaimeoirí i scoileanna a mhúineann trí mheán an Bhéarla, éiríonn le foghlaimeoirí lán-Ghaeilge caighdeán níos airde a bhaint amach sa Ghaeilge (Harris, Forde, Nic Fhearaile \& O’ Gorman, 2006), agus torthaí atá thar an meán náisiúnta a bhaint amach, sa léitheoireacht Bhéarla agus sa Mhatamaitic (Gileece, Shiel, 
Clerkin \& Millar, 2012). Tá taithí ag múinteoirí lán-Ghaeilge ar ábhair a theagasc trí mheán an dara teanga (An Ghaeilge), agus tá taithí ag páistí lán-Ghaeilge ar ábhair a fhoghlaim trí mheán an dara teanga (An Ghaeilge). D'fhéadfaí rath na foghlama teanga sna scoileanna lánGhaeilge a leathnú amach chuig an tríu teanga, dá múinfí ábhar nó gné d'ábhar tríd an tríu teanga (An Foras Patrúnachta, 2018).

Tá cáil ar an bhFoghlaim Chomhtháite Ábhar agus Teangacha (FCÁT, nó Content and Language Integrated Learning, CLIL) ar fud na hEorpa, mar chur chuige atá éifeachtach leis an dara teanga nó teanga bhreise a theagasc (Coyle, Hood \& Marsh, 2010). Is scáth-théarma é FCÁT a chuimsíonn iliomad múnlaí (Ioannou-Georgio \& Pavlou, 2011; Lo, 2020; Mehisto, Marsh \& Frigols, 2008) ar an gcontanam den fhoghlaim chomhtháite ábhar agus teangacha. Is iomaí agus is éagsúil iad na difríochtaí idir cláir FCÁT, maidir le spriocanna clár, sprioctheanga an teagaisc agus stádas na teanga sin, próifíleanna múinteoirí agus mac léinn, polasaithe oideachasúla, dearadh curaclaim agus cleachtais oideolaíochta (Lo, 2020; Lyster \& Ballinger, 2011). Ar lámh amháin, tá cáineadh déanta ar FCÁT sa litríocht de bharr easpa soiléireachta sa téarmaíocht a úsáidtear (Paran, 2013), ar an lámh eile, tugtar ardmholadh do FCÁT, mar gur cur chuige solúbtha é a fhreagraíonn d'éagsúlacht teangacha (Pérez-Cañado, 2017). Tá FCÁT ag teacht le múnlaí tumoideachais, mar gurb é suíomh foghlama an ábhair a spreagann próiseas sealbhaithe na teanga, trí dheiseanna ionchuir, deiseanna idirghníomhaithe agus deiseanna aschuir a chur ar fáil d'fhoghlaimeoirí sa sprioctheanga (Dalton-Puffer, 2011). Áitítear gur chóir múnlaí éagsúla de FCÁT a fhorbairt de réir mar a oireann do gach sainchomhthéacs foghlama (Coyle, 2007; Pérez-Cañado, 2017). Agus cláir nua FCÁT á bhforbairt, moltar go ndéanfaí taighde le linn na gcéimeanna tosaigh d'fhonn teacht roimh na dúshláin agus cur le héifeachtacht na gclár ón tús (Sylvén, 2013). Tá sé mar aidhm ag an alt seo, tús a chur le hiniúchadh a dhéanamh ar na féidearthachtaí a bhainfeadh le FCÁT a chur i bhfeidhm tríd an tríú teanga i mbunscoileanna lán-Ghaeilge.

Léiríonn mórbheartas an rialtais do theagasc teangacha iasachta sa chóras oideachais in Éirinn (An Roinn Oideachais agus Scileanna, 2017), chomh tábhachtach is atá foghlaim teangacha iasachta ‘do leas cultúrtha, leas sóisialta agus eacnamaíoch na hÉireann' (lch. 20). Tá sé mar sprioc ag an mbeartas ról an chórais oideachais a neartú maidir le méadú rannpháirtíochta agus cumais i dteangacha iasachta. Tá FCÁT molta go sonrach sa bheartas mar chur chuige leis an nGaeilge a chur chun cinn i scoileanna arb é an Béarla an meán teagaisc ag an mbunleibhéal, agus tá tograí píolótacha ar FCÁT trí Ghaeilge molta don idirbhliain agus san ardoideachas. 
Cé nach luaitear FCÁT trí theanga iasachtach ag an mbunleibhéal go sonrach sa bheartas, moltar go ndéanfaí athbhreithniú ar na féidearthachtaí a bhaineann le teagasc teanga iasachta sna hardranganna bunscoile. Bhronnfadh FCÁT trí theanga iasachtach sna scoileanna lánGhaeilge deis ar pháistí dul i dtaithí ar an tríu teanga ag leibhéal na bunscoile. Rachadh an cleachtas seo i dtreo fhís an Aontais Eorpaigh, a mholann gur chóir máthairtheanga agus dhá theanga eile a bheith ar a dtoil gach saoránach san Aontas Eorpach (Dalton-Puffer, 2007). Mhol an Phróifíl Bheartas Oideachais Teanga, Éire (Comhairle na hEorpa, 2008), do shaoránaigh na hÉireann bogadh ar aghaidh ón bhfís de shochaí dhátheangach, agus sochaí ilteangach a chothú, a aithníonn an luach atá le foghlaim agus úsáid teanga iasachta amháin nó níos mó sa chóras oideachais agus ar feadh an oideachais fhad shaoil.

Déantar cur síos san alt seo ar thaithí cúigear múinteoirí agus príomhoide amháin ar FCÁT a chur i bhfeidhm tríd an tríu teanga i mbunscoileanna lán-Ghaeilge. Is scéim phíolótach ar scála beag a bhí sa taighde seo agus ghlac sé bhunscoil páirt sa scéim. Chomhtháthaigh na múinteoirí teanga Eorpach (an Fhraincis nó an Ghearmáinis), leis an bhfoghlaim ábhair in ábhar scoile amháin (Ealaín/Corpoideachas/Ceol), i rith na scoilbhliana. Léirítear sa litríocht go ndíríonn formhór an chleachtais agus taighde ar FCÁT, ar mhúineadh ábhar trí mheán an Bhéarla (Dalton-Puffer, 2011), agus ar fhoghlaimeoirí ag an meánleibhéal nó ag an tríú leibhéal (Ioannou-Georgio \& Pavlou, 2011). Léirigh tuarascáil Eurydice (Eurydice, 2006), nach raibh soláthar trítheangach de FCÁT le fáil, ach i seacht dtír san Eoraip: An Eastóin, an Spáinn, Latvia, Luxembourg, an Ísiltír, an Ostair agus an tSualainn. Áitítear go dteastaíonn breis taighde agus breis fhorbartha ar FCÁT trí réimse níos leithne teangacha (Dalton-Puffer, 2011), ag leibhéal na bunscoile (Ioannou-Georgio \& Pavlou, 2011), agus i suíomhanna trítheangacha (Merino \& Lasagabaster, 2018). Is ábhar taighde nuálach a bhí sa scéim phíolótach áirithe seo, mar go raibh FCÁT curtha i bhfeidhm tríd an tríu teanga le foghlaimeoirí óga i suíomh an tumoideachais lán-Ghaeilge. Is éard atá san alt seo ná anailís agus plé ar shonraí cáilíochtúla a bailíodh ó cúigear múinteoirí agus príomhoide amháin a bhí páirteach sa scéim phíolótach. Is díol spéise é an t-eolas seo mar go dtugtar spléachadh dúinn ar chuid de na féidearthachtaí agus na dúshláin a bheadh roimh bhunscoileanna lán-Ghaeilge, dá mbeadh FCÁT le cur i bhfeidhm ar bhonn níos leithne amach anseo. Ní mór a shoiléiriú gur tuairisciú ar eispéiris na múinteoirí amháin, dar leis na múinteoirí féin, atá san alt seo. Ní léargas é ar a (mhí)éifeachtaí is atá FCÁT i gcomhthéacs na mbunscoileanna lán-Ghaeilge. Cé gur miontaighde atá sa taighde seo, tá súil ag an údar go spreagfaidh an t-alt dioscúrsa, machnamh agus breis taighde ar FCÁT tríd an tríú teanga sna bunscoileanna lán-Ghaeilge. 
D'fhonn an tionscadal taighde seo a lonnú i gcomhthéacs déantar athbhreithniú gonta ar an litríocht ábhartha anois faoi na mórthéamaí seo a leanas: saineolas ábhair oideolaíoch FCÁT, forbairt ghairmiúil múinteoirí do FCÁT, FCÁT ag leibhéal na bunscoile agus luath-fhoghlaim teangacha iasachta.

\section{Léirmheas Litríochta}

\subsection{Saineolas Ábhair Oideolaíoch FCÁT}

Tá an-cháil ar an gcoincheap ar ghlaoigh Shulman $(1986,1987)$ eolas ábhair oideolaíoch air, sa litríocht ar oideachas múinteoirí le tamall de bhlianta anuas. Is éard is brí le heolas ábhair oideolaíoch ná an scil trína n-athraíonn múinteoirí an t-eolas ábhair atá acu go foirm atá fóirsteanach do theagasc agus d'fhoghlaim an ábhair. Teastaíonn eolas ábhair oideolaíoch ar leith ó mhúinteoirí FCÁT mar gur gá dóibh an t-eolas ábhair a sholáthar ar bhealach sothuigthe trí theanga nach máthairtheanga na bhfoghlaimeoirí í (Coyle, Hood \& Marsh, 2010). Ag tógáil ar shaothar Ball, Thames \& Phelp (2007) a rinne forleathnú ar shaothair Shulman i gcomhthéacs na matamaitice, agus ar shaothar Freeman, Katz, García Gómez \& Burns (2015) a dhírigh ar shaineolas teangeolaíochta múinteoirí teanga, tá fráma coincheapúil deartha ag Morton $(2016,2017)$ le léargas a thabhairt ar eolas ábhar oideolaíoch múinteoirí FCÁT.

Tá ceithre chuid idirghaolmhara léirithe i bhfráma coincheapúil Morton $(2016,2017)$ d'eolas ábhar oideolaíoch múinteoirí FCÁT is iad sin: (i) buneolas teangeolaíochta don teagasc ábharbhunaithe (BE-TÁ), (ii) saineolas teangeolaíochta don teagasc ábharbhunaithe (SETÁ), (iii) eolas faoin teanga agus foghlaimeoirí (ET-F), agus (iv) eolas faoin teanga agus teagasc (ET-T). Is é is brí le BE-TÁ, ná inniúlacht sa sprioctheanga a áiríonn eolas cumarsáideach ar an teanga agus eolas ar an teanga acadúil araon, mar sin cuimsíonn BE-TÁ, bunscileanna cumarsáide idirphearsanta (BICS) agus inniúlacht ar an teanga chognaíoch agus acadúil (CALP) mar atá léirithe i múnla teoiriciúil Cummins (2000). Tá eolas intuigthe/gnásúil ar an teanga, chomh maith le heolas follasach/ fógrach sa réimse BE-TÁ. Ní mór a aithint go bhféadfadh BE-TÁ a bheith ag úsáideoirí inniúla teanga, nach múinteoirí teanga nó ábhair iad. Is éard atá gceist le SE-TÁ ná eolas teangeolaíochta a bhfuil dlúthnasc aige le torthaí foghlama an ábhair. Is cineál eolais é, a chabhraíonn le múinteoirí an tsainteanga agus na struchtúir atá de dhíth le dul i mbun foghlama an ábhair a aithint, agus an t-eolas ábhair a bhriseadh síos i dtéarmaí teangeolaíochta d'fhoghlaimeoirí. Nascann ET-F an t-eolas teangeolaíochta atá ag múinteoirí leis an sainaithne atá acu ar na foghlaimeoirí atá á dteagasc acu. Tarraingíonn ET-F aird ar an scil trína gcuireann múinteoirí an teanga in oiriúint do riachtanais foghlaimeoirí ar 
leith agus faoi mar a chuireann siad creat tacaíochta teanga ar fáil dóibh. Baineann ET-T, leis an réimse scileanna agus straitéisí oideolaíocha a chuireann múinteoirí i bhfeidhm le torthaí foghlama ábhair agus teanga a chomhtháthú ar bhealach bríoch agus comhlántach.

\subsection{Forbairt Ghairmiúil do FCÁT}

Léirítear go bhfuil dlúthcheangal idir éifeachtacht múinteoirí agus gnóthachtáil foghlaimeoirí sa chóras oideachais (Darling Hammond, 2000). Aithnítear go bhfuil géarghá forbairt ghairmiúil a chur ar fáil do mhúinteoirí thar an gcontanam den fhoghlaim chomhtháite ábhar agus teangacha le dea-chleachtas a nochtadh (Lo, 2020; Lyster \& Ballinger, 2011; Mac Dougald, 2019; Ó Ceallaigh, Ó Laoire, \& Uí Chonghaile, 2019). Cothaíonn an réimse fairsing cur chuige atá i gceist le FCÁT dúshlán do sholáthar na forbartha gairmiúla (Frigols Martin, 2011). Ar aon dul le Comhchreat Tagartha na hEorpa um Theangacha (Comhairle na hEorpa, 2001; 2018), tá Creat Eorpach um Oideachas Múinteoirí CLIL (Frigols Martín, Marsh, Mehisto, Wolff, 2011) curtha ar fáil ag Comhairle na hEorpa mar uirlis choincheapúil le tacú le forbairt clár forbartha gairmiúla FCÁT agus mar uirlis athmhachnaimh do mhúinteoirí. Leagtar síos an t-eolas agus na buninniúlachtaí gairmiúla a theastaíonn ó mhúinteoirí le FCÁT a chur i bhfeidhm ar bhealach éifeachtach agus comhlántach sa chreat. Níl an creat sainoiriúnaithe d'aon chomhthéacs ar leith, ach cuirtear treoir ar fáil atá fréamhaithe i mbunphrionsabail de dhea-chleachtas FCÁT a d'fhéadfaí a chur in oiriúint d'aon suíomh ar leith.

Moltar cur chuige ón mbonn aníos agus cláir forbartha gairmiúla á bhforbairt sa chaoi is go mbeadh spriocanna an chláir idirbheartaithe idir scoileanna, múinteoirí agus údaráis curaclaim (Bangeas, 2012; Mac Dougald, 2019). Molann Mac Dougald (2019) nár mhór go mbeadh forbairt ghairmiúil FCÁT sainoiriúnaithe do gach comhthéacs foghlama agus dírithe ar: mhórchuspóirí an chláir (atá idirbheartaithe ag páirtithe leasmhara), sainriachtanais múinteoirí agus cleachtas sa seomra ranga. Moltar sainriachtanais oideolaíocha múinteoirí a chur ag croílár na forbartha gairmiúla, agus nárbh mhór múinteoirí a spreagadh le spriocanna foghlama a leagan síos dóibh féin ar bhonn leanúnach.

\subsection{FCÁT ag an mbunleibhéal}

Is díol spéise é an tionscadal ProCLIL (Ioannou-Georgiou \& Pavlou, 2011) a reáchtáil an Cumann Eorpach faoi scáth an chláir Comenius don alt seo. Bhailigh an tionscadal eolas ar FCÁT san oideachas luath-óige agus ag an mbunleibhéal i gcúig thír Eorpacha thar thréimhse 
trí bliana. Bhí sé mar aidhm ag an tionscadal treoirlínte a dhearadh do mhúinteoirí agus scoileanna, a léireodh dea-chleachtas agus a thacódh le cur i bhfeidhm FCÁT ag an mbunleibhéal. De réir na dtreoirlínte a dearadh, is cur chuige de réir a chéile a mholtar d'fhoghlaimeoirí FCÁT ag an mbunleibhéal. Moltar tosú ar úsáid na teanga go neamhfhoirmiúil i rith an lae, agus cuid den cheacht ábhair (seachas an ceacht ar fad), a mhúineadh trí mheán na sprioctheanga. Moltar gur gá meascán den sprioctheanga agus de chéad teanga na scoile a úsáid i gceachtanna FCÁT ag an mbunleibhéal, d'fhonn tacú le foghlaimeoirí óga, torthaí foghlama ábhar an churaclaim a bhaint amach sa dara teanga (Ioannou-Georgio \& Pavlou, 2011). Léirigh an tionscadal ProCLIL, go raibh sainscileanna oideolaíochta ag teastáil ó mhúinteoirí FCÁT le creat tacaíochta foghlama a chur ar fáil d'fhoghlaimeoirí óga, a éascaíonn foghlaim na sprioctheanga, foghlaim an ábhair, agus foghlaim an ábhair trí mheán na sprioctheanga dóibh (Ioannou-Georgio \& Pavlou, 2011). D’fhéadfaí a áitiú, go mbeadh saineolas ábhair oideolaíoch don fhoghlaim chomhtháite ábhar agus teangacha forbartha ag múinteoirí lán-Ghaeilge agus taithí laethúil acu ar theagasc ábhar trí mheán na Gaeilge d'fhoghlaimeoirí Gaeilge. Ba iad na príomhdhúshláin a tháinig chun cinn maidir le cur bhfeidhm FCÁT ag an mbunleibhéal san Eoraip ná: inniúlacht teanga na múinteoirí, easpa forbartha gairmiúla, ganntanas áiseanna teagaisc, mar aon leis an am agus dua breise a bhain le ceachtanna FCÁT a réiteach agus a theagasc i gcomparáid le ceachtanna ábhar i gcéad teanga na scoile (Ioannou-Georgiou \& Pavlou, 2011).

\subsection{Luath-Fhoghlaim Teangacha Iasachta}

Cé nach gcinntíonn foghlaim teangacha iasachta sa luath-fhoghlaim go mbeidh rath ar an bhfoghlaim teanga (Harris \& Ó Duibhir, 2011), d'fhéadfadh foghlaim teangacha iasachta sa bhunscoil, foghlaimeoirí óga a réiteach le staidéar níos oilte a dhéanamh ar theangacha iasachta ag an meánleibhéal (Hawkins, 2005). Shamhlaigh Hawkins (2005), foghlaim teangacha ar scoil mar dhá chuid de phrintíseacht. Mhol sé gur cheart díriú ar spriocanna oideachasúla foghlama teanga ag an mbunleibhéal, agus ar spriocanna feidhmiúla foghlama teanga ag an meánleibhéal. Is é is brí le spriocanna oideachasúla foghlama teanga, ná spreagadh na feasachta teanga agus forbairt scileanna le teangacha a fhoghlaim - 'ag foghlaim conas foghlaim'. Cuimsíonn an fheasacht teanga forbairt scileanna meititheangeolaíocha le labhairt faoi theangacha, chomh maith le páistí a spreagadh le haird a thabhairt ar na difríochtaí agus ar na cosúlachtaí idir teangacha. Mhol Hawkins (2005), teagasc an cheoil ach go háirithe, mar chur chuige le hoiliúint a chur ar chluas foghlaimeoirí óga le teangacha iasachta a fhoghlaim. Cuireann spriocanna feidhmiúla foghlama teanga béim ar theanga a fhoghlaim d'fhonn cuspóirí 
sonracha a bhaint amach nuair atá an foghlaimeoir níos aibí, m.sh., cáilíocht a bhaint amach i scrúdú nó cumarsáid a dhéanamh i suíomh ar leith. Tagann na cláir fheasachta teanga a reáchtáladh ag an mbunleibhéal i Sasana (Barton, Bragg \& Serratrice, 2009; Jones, Barnes \& Hunt, 2005) nuair a bhronnadh deis ar fhoghlaimeoirí teangacha éagsúla a fhoghlaim thar thréimhsí gairide ama le fís Hawkins. Cuireadh béim sna cláir ar spéis a chothú i bhfoghlaim teangacha, seachas ar leibhéil áirithe inniúlacht a bhaint amach in aon teanga ar leith Léiríonn anailís ar na cláir gur éirigh leo, feasacht teanga a chothú, agus inspreagadh foghlaimeoirí a mhéadú le teangacha iasachtacha a fhoghlaim. Aithnítear go n-oireann cláir fheasachta teanga, ach go háirithe, do mhúinteoirí nach sainmhúinteoirí teanga iad, cur i gcás múinteoirí bunscoile (Barton, Bragg \& Serratrice, 2009; Jones, Barnes \& Hunt, 2005).

\section{Modheolaíocht}

Bhí deis ag na múinteoirí a ghlac páirt sa scéim phíolótach FCÁT, freastal ar dhá cheardlann forbartha gairmiúla FCÁT i rith na scoilbhliana 2017/2018. Mhair na ceardlanna ar feadh an lae scoile agus díríodh ar bhunphrionsabail teoiriciúla agus oideolaíochta FCÁT, straitéisí oideolaíochta FCÁT chomh maith le pleanáil do chomhtháthú an ábhair agus na teanga ar bhealach comhlántach lena linn. Bhí deis ag múinteoirí labhairt lena chéile agus a gcuid tuairimí agus taithí a roinnt. Is ball den tseirbhís um fhorbartha gairmiúla do mhúinteoirí a bhí mar áisitheoir sa chéad cheardlann, agus b'oideoir múinteoirí i dTeagasc na Gaeilge in Institiúid Oideachais, Ollscoil Chathair Bhaile Átha Cliath (OCBÁC) a bhí mar áisitheoir sa dara ceardlann.

Bailíodh eolas faoi eispéiris na múinteoirí i mí na Bealtaine 2019, tar éis dóibh naoi mí a chaitheamh i mbun na scéime píolótaí. Fuair údar an ailt cead eitice ó Choiste Eitice (OCBÁC) sonraí a bhailiú ó na múinteoirí. Tugadh ráiteas i bhfriotal simplí, a rinne cur síos ar chuspóirí an taighde, do na múinteoirí agus shíníodar ar fad foirm i ndáil le toiliú feasach. Deimhníodh nach mbainfí úsáid as torthaí an taighde ach amháin ar son na gcuspóirí a aontaíodh. Úsáidtear ainmneacha bréige sa chur síos ar an taighde le rúndacht na múinteoirí agus na scoileanna a chosaint. Bailíodh eolas faoi chúlra teanga agus teagaisc na múinteoirí, mar aon lena dtuairimí ginearálta faoi bhuanna agus dúshláin na scéime píolótaí i gceistneoir. Tá cur síos ar thaithí teagaisc na múinteoirí, an sprioctheanga a roghnaigh siad, chomh maith lena n-uascháilíocht sa sprioctheanga léirithe i dTábla 1. Léiríonn Tábla 2 na ranganna agus na hábhair a mhúin na múinteoirí le linn na scéime píolótaí. 


\begin{tabular}{|l|l|l|l|}
\hline $\begin{array}{l}\text { Ainm an } \\
\text { Mhúinteora }\end{array}$ & Blianta i mbun Teagaisc & Sprioctheanga & Uascháilíocht sa Sprioctheanga \\
\hline Vicky & $5-10$ mbliana & Fraincis & Céim Ollscoile \\
\hline Cáit & $5-10$ mbliana & Gearmáinis & Sprachdiplom $1 \& 2$ \\
\hline Laura & $<5$ bliana & Fraincis & Ardteist \\
\hline Donna & $10-20$ bliain & Fraincis & Céim Ollscoile \\
\hline Niamh & $<5$ bliana & Fraincis & Céim Ollscoile \\
\hline
\end{tabular}

Tábla 1: Blianta i mbun Teagaisc sa Bhunscoil agus Uascháilíocht sa Sprioctheanga

\begin{tabular}{|l|l|l|}
\hline $\begin{array}{l}\text { Ainm an } \\
\text { Mhúinteora }\end{array}$ & Rang & \\
\hline Vicky & Naíonáin Shóisearacha & Corpoideachas \\
\hline Cáit & Rang a hAon & Ealaín \& Ceol \\
\hline Laura & Naíonáin Shinsearacha & Ealaín \\
\hline Donna & Naíonáin Shinsearacha & Amhránaíocht \\
\hline Niamh & Rang a hAon & Ealaín \\
\hline
\end{tabular}

Tábla 2: Ranganna agus Ábhair a mhúin na múinteoirí

Rinneadh anailís ábhair ar na sonraí a bhí sna ceistneoirí agus bhí deis ag an taighdeoir cíoradh níos grinne a dhéanamh ar na freagraí a tháinig chun cinn sa cheistneoir le linn an ghrúpa fócais. Baineann grúpa fócais le "generating hypotheses that derive from the insights and data from the group" (Cohen, Manion \& Morrison, 2017, lch. 532). Cuireadh tús leis an ngrúpa fócais le ceisteanna leathana chun na rannpháirtithe a chur ar a suaimhneas, agus d'éirigh na ceisteanna níos dírithe lena linn. Rinneadh taifead fuaime ar an ngrúpa fócais agus tugadh faoi anailís a dhéanamh ar bhonn téamúil (Braun \& Clarke, 2006) ar na sonraí a bailíodh. Leanadh na sé chéim anailíse seo: (i) eolas a chur ar na sonraí trí éisteacht agus athéisteacht leis an taifead fuaime; (ii) fráma códaithe a dhearadh as pointí suimiúla a tháinig chun cinn sa ghrúpa fócais; (iii) rangú a dhéanamh ar na cóid de réir téamaí; (iv) athbhreithniú a dhéanamh ar na téamaí d'fhonn iad a bheachtú; (v) sainmhíniú a thabhairt ar na téamaí; (vi) sintéisiú agus infeireas a bhaint as torthaí an taighde. 
Déantar cur síos anois ar na príomhthéamaí a tháinig chun cinn le linn an ghrúpa fócais. Faightear spléachadh ar chuid de na buanna agus na dúshláin fhéideartha a bhainfeadh le FCÁT a chur i bhfeidhm tríd an tríu teanga i mbunscoileanna lán-Ghaeilge ó fhéintuarascáil na múinteoirí. Ba iad na téamaí ba mhó a d'eascair ón anailís ná: inspreagadh agus dearcthaí dearfacha na múinteoirí; cleachtais éagsúla FCÁT; buanna agus dúshláin na scéime píolótaí; mar aon le fís do FCÁT agus forbairt na físe sin i sainchomhthéacs na mbunscoileanna-lán Ghaeilge in Éirinn.

\section{Torthaí agus Plé}

\subsection{Inspreagadh agus Dearcthaí Dearfacha na Múinteoirí}

Bhí na múinteoirí ar aon tuairim gurb é a dtaithí féin mar mhúinteoirí lán-Ghaeilge a thug spreagadh agus muinín dóibh páirt a ghlacadh sa scéim phíolótach. Bhí na múinteoirí ar aon intinn go mbaineann leas le foghlaim teangacha, agus léirigh siad ar fad dearcadh dearfach i leith foghlaim ábhar trí mheán an dara teanga. Tagann dearcthaí na múinteoirí leis an litríocht ar oideachas múinteoirí a aibhsíonn go n-imríonn taithí phearsanta an mhúinteora, tionchar ar chognaíocht agus ar chleachtas an mhúinteora sa rang (Freeman, 1989; Kennedy, 1991). Nocht cuid na múinteoirí an tuairim go mbeadh an suíomh lán-Ghaeilge an-bháúil do theagasc tríd an tríú teanga.

Feicim sna naíonáin - cé chomh nádúrtha is a phiocann siad suas an teanga. (Vicky)

Chomh maith lena dtaithí ar theagasc tríd an dara teanga, nasc Cáit agus Nuala a dtaithí féin, mar iar-scolairí lán-Ghaeilge lena n-inspreagadh tabhairt faoi FCÁT.

$$
\begin{aligned}
& \text { Mé féin d'fhreastalaíos ar bhunscoil agus meánscoil trí mheán na Gaeilge. Agus } \\
& \text { ceapaim féin nach bhfuil aon tslí nach bhfoghlaimeodh na páistí tríd an tríu teanga } \\
& \text { nó tríd an gceathrú teanga go háirithe má tá siad i suíomh le tumoideachas. (Nuala) }
\end{aligned}
$$

Tá sé léirithe sa taighde go dteastaíonn saineolas ábhar oideolaíoch ar leith ó mhúinteoirí FCÁT (Frigols Martin et al., 2011; Morton, 2016, 2017). D'fhéadfaí a léamh ar na torthaí gur thug an t-eolas ábhar oideolaíoch atá forbartha ag na múinteoirí ó bheith ag saothrú sa suíomh lánGhaeilge, chomh maith lena dtaithí mar fhoghlaimeoirí lán-Ghaeilge, muinín dóibh tabhairt faoin teagasc comhtháite ábhar agus teanga leis an tríú teanga. 
Tháinig sé chun solais go raibh tuairimí cuid de na múinteoirí ag teacht leis an bhfís Eorpach d'fhorbairt an ilteangachais tríd an gcóras oideachais (An Roinn Oideachais \& Scileanna, 2017; Comhairle na hEorpa, 2008). Mhínigh Vicky toisc go raibh sí ag obair i scoil ilchreidmheach, gur thaitin an fhís de scoil ilteangach leis an bhfoireann. Léirigh Donna an tuairim go bhféadfadh teagasc teangacha Eorpacha sa bhunscoil cabhrú leis na páistí nasc níos mó a dhéanamh leis an Eoraip.

Tuigim gur rud ana-mhaith é dóibh ó thaobh iad a mhothú níos Eorpaí. In Éirinn tá sé deacair go leor mothú mar chuid den Eoraip. Tá siad i bhfad níos ceangailte má tá an tríú teanga acu. (Donna)

\subsection{Cleachtais Éagsúla FCÁT}

Tháinig sé chun cinn sa ghrúpa fócais gur cuireadh múnlaí éagsúla FCÁT i bhfeidhm sna scoileanna. D'éirigh le scoil Vicky cur chuige uile scoile FCÁT a chur i bhfeidhm mar go raibh Fraincis ag leibhéal na hardteistiméireachta ag gach múinteoir ranga, agus iad ar fad toilteanach páirt a ghlacadh sa scéim. Mhínigh Vicky gur roghnaigh an fhoireann an corpoideachas mar ábhar mar gheall gur ábhar gníomhach é atá suite i suíomh taobh amuigh den seomra ranga (Lamb \& King, 2020). Mar gheall gur chlúdaigh gach rang sa scoil an snáithe céanna den churaclam corpoideachais ag an aon am amháin, bhain bua leis seo maidir le pleanáil foirne don chomhtháthú ábhar agus teanga. Toisc go raibh an Fhraincis mar sprioctheanga i ngach rang, d'éirigh leis an bhfoireann an Fhraincis a chomhtháthú le gníomhaíochtaí neamhfhoirmiúla scoile chomh maith m.sh, glaoch an rolla, úsáid na Fraincise ag an tionóil srl. Tá comhtháthú neamhfhoirmiúil na sprioctheanga molta mar chur chuige le próifíl na sprioctheanga a ardú agus le cabhrú le foghlaimeoirí óga dul i dtaithí ar an teanga ag leibhéal na bunscoile (Ioannou-Georgiou \& Pavlou, 2011). Ní raibh ach rang amháin páirteach sa tionscadal sa ceithre scoil eile. Ba léir ó aiseolas an ghrúpa fócais, go bhfuil cur chuige uile scoile níos indéanta nuair a bhíonn leibhéal áirithe inniúlachta bainte amach ag gach múinteoir sa sprioctheanga chéanna.

Léiríodh cleachtais éagsúla maidir le húsáid na sprioctheanga sna ranganna chomh maith. Chomhtháthaigh Laura agus Vicky, an Fhraincis leis na gníomhaíochta foghlama sa rang, agus mhúin siad cuid den cheacht ábhair trí mheán na sprioctheanga. I rang Vicky, mar shampla, rinne siad an téamh suas sa chorpoideachas trí Fhraincis, agus i rang Lauren, úsáideadh ainmneacha na ndathanna i bhFraincis. Mhínigh Vicky agus Lauren gur oir cur chuige de réir 
a chéile leo, mar gheall ar a bhféinbhraistint faoina n-inniúlacht féin sa sprioctheanga. Bheartaigh Nuala agus Cáit an ceacht ar fad a mhúineadh trí mheán na sprioctheanga, de bharr go raibh an muinín acu beirt sin a dhéanamh. Ag eascairt ó eispéiris na múinteoirí, d'fhéadfaí a áitiú go n-imríonn inniúlacht an mhúinteora tionchar ar an méid den sprioctheanga a úsáidtear sa rang. Dá mbeadh forbairt ghairmiúil ar FCÁT trí theanga Eorpach le soláthar do mhúinteoirí, agus saináiseanna le dearadh, ní mór tabhairt san áireamh, go mbeadh teangacha éagsúla Eorpacha ar eolas ag múinteoirí bunscoile, agus go mbeadh raon leathan inniúlachta i gceist. Ní mór a aithint fosta, nach bhfuil eolas ar an tríú teanga mar choinníoll iontrála ar chláir oideachais mhúinteoirí agus nach gá go mbeadh eolas ar an tríu teanga ag gach múinteoir lán-Ghaeilge. Toisc go raibh cleachtais éagsúla agus teangacha éagsúla i gceist sna scoileanna agus idir scoileanna, is léir nár mhór do chláir forbartha gairmiúla a bheith sainoiriúnaithe do scoileanna agus múinteoirí ar leith (Banegas, 2012; Mac Dougald, 2019).

Ba dhíol spéise é go raibh Donna airdeallach nach gcuirfeadh teagasc an tríu teanga isteach ar an tumoideachas lán-Ghaeilge agus páistí óga ag sealbhú na Gaeilge sna Naíonáin Shinsearacha. In ionad ceachtanna foirmiúla a theagasc trí Fhraincis, bheartaigh Donna ar roinnt amhrán i bhFraincis a chur ar siúl do na páistí i rith an lae. Mhol Donna agus Katie beirt, an amhránaíocht leis an tríú teanga a chur chun cinn. Dar leo, bhain bua leis an gceol toisc gur féidir é a chomhtháthú le gníomhaíochtaí an lae, agus go bhfuil sé éasca teacht ar amhráin ar líne. D’aithin siad éifeachtacht an cheoil le fuaimeanna na teanga a chur ar chluas na bpáistí agus mar chur chuige le foghlaim na teanga a chur chun cinn go neamhfhoirmiúil. Tagann an méid a bhí le rá acu faoi oiriúnacht an cheoil do theagasc teanga sa bhunscoil le saothar Hawkins (2005).

Bhí muid ag iarraidh rud beag a dhéanamh gach lá agus b 'shin an ceol. (Cáit)

Phiocadar suas é i bhfad níos nádúrtha ná dá mbeimis ag déanamh ábhair. (Diane)

\subsection{Buanna an tionscadail}

Luaigh na múinteoirí ar fad taitneamh na bpáistí, agus páistí ag úsáid na sprioctheanga mar bhuanna a bhain leis an tionscadal. Bhí na múinteoirí ar aon tuairim gur ghlac na páistí go dearfach leis an gcur chuige FCÁT. D’fhéadfaí a áitiú go trialach, go mbeadh foghlaimeoirí lán-Ghaeilge an-oscailte do FCÁT agus taithí acu ar chóras an tumoideachais, agus ar fhoghlaim trí mheán an dara teanga cheana féin. Ba léir ó fhianaise na múinteoirí go raibh tús 
curtha le próiseas foghlama an tríú teanga. Thuairiscigh Nuala go raibh iontas uirthi na páistí a chloisteáil ag úsáid focal i bhFraincis anseo agus ansiúd i rith an lae. Mhínigh sí gurb é an foclóir agus na heiseamláirí ba choitianta a chuala na páistí ó sheachtain go seachtain, a bhí in úsáid acu.

Bíonn ionadh ort nuair a thagann siad amach le focail. (Lauren)

Léirigh na múinteoirí go raibh ionchais réadúla acu maidir le torthaí foghlama teanga na bpáistí sa tríu teanga agus gur chabhraigh an dearcadh seo leo atmaisféar dearfach foghlama a chothú sa rang:

Más rud é gur focal nó dhó a bhí ann bhí gach duine chomh sásta leis sin. (Lauren)

Caithfidh tú tosú le rud éigin, áit éigin ag am éigin' (Niamh)

Cé go gcáintear simpliú na dtorthaí foghlama ábhar i gcuid den litríocht ar FCÁT (Costa \& d'Angelo, 2011), ní raibh na múinteoirí den tuairim gur chothaigh an cur chuige FCÁT míbhuntáiste ar bith do bhaint amach na dtorthaí foghlama ábhar. D'fhéadfaí a rá go trialach go mbeadh saineolas ábhar oideolaíoch a bhaineann le comhtháthú ábhar agus teanga forbartha (Morton, 2016, 2017) ag múinteoirí lán-Ghaeilge ó bheith ag saothrú sa suíomh lán-Ghaeilge agus go mbeadh an saineolas seo ina cuidiú acu dá mbeadh FCÁT le cur i bhfeidhm FCÁT tríd an tríú teanga.

\subsection{Dúshláin}

B’ionann na dúshláin a bhí ag na múinteoirí a ghlac páirt sa tionscadal seo, agus na príomhdhúshláin a tháinig chun cinn do mhúinteoirí bunscoile sa tionscadal PROCLIL (Ioannou-Georgiou \& Pavlou, 2011). Ba iad na príomhdhúshláin a thuairiscigh na múinteoirí ná, a n-inniúlacht féin sa sprioctheanga, easpa forbartha gairmiúla, ganntanas líonraí forbartha agus ganntanas saináiseanna de FCÁT.

Ba léir gur chuir easpa muiníne na múinteoirí maidir lena n-inniúlacht féin sa sprioctheanga isteach ar a bhféinmhuinín sa rang. Dúirt Donna go mbeadh easpa muiníne uirthi ó thaobh na foghraíochta de, dá mbeadh Francach istigh sa rang ag éisteacht léi. Mhínigh Laura nach raibh 
sí cinnte an raibh cuid den fhoclóir á fhuaimniú i gceart aici agus go dteastódh tacaíocht ó mhúinteoirí lena n-inniúlacht a ardú sa sprioctheanga.

Tá sé an-deacair nuair a dhéanann tú botún, níl a fhios agat an bhfuil tú ag úsáid Fraincis cheart nó atá Fraincis mícheart agus muna bhfuil éinne ann le ceist a chur orthu ag deireadh an lae. (Donna)

Mhínigh na múinteoirí cé go raibh staidéar déanta acu ar an sprioctheanga sa mheánscoil, nach raibh an tsainteanga a theastaíonn don seomra ranga ar a dtoil acu, m.sh, teanga maidir le treoracha a thabhairt agus gnáthchumarsáid a dhéanamh leis na páistí. Is léir go n-éilíonn suíomh an tseomra ranga (Freeman et al., 2015) agus FCÁT saineolas teangeolaíochta ar leith ó mhúinteoirí (Morton, 2016, 2017).

Níl an teanga sin agat agus tú ag fágáil na hardteistimeireachta fiú má tá scrúdú béil iontach déanta agat. (Donna)

D’aithin na múinteoirí nasc coincheapúil idir úsáid na Gaeilge go neamhfhoirmiúil i scoileanna arb é an Bhéarla an meán teagaisc, agus úsáid neamhfhoirmiúil an tríu teanga sna scoileanna lán-Ghaeilge. Mhol na múinteoirí go mbeadh gluais teanga sa sprioctheanga ina áis iontach do mhúinteoirí. Mhíníodar freisin gur bhain dúshlán le háiseanna teagaisc a mheas mar gheall ar laigí inniúlachta sa sprioctheanga. Is féidir a léamh ar na torthaí seo gur mhothaigh na múinteoirí go raibh easnaimh acu sa tsainteangeolaíocht a bhaineann le FCÁT (Morton, 2016, 2017), agus go gcuirfeadh na heasnaimh seo srian lena n-éifeachtacht mar mhúinteoirí FCÁT.

Nocht na múinteoirí an tuairim go raibh sé deacair a bheith ag obair ina n-aonair agus gur bhain an-dua le háiseanna feiliúnacha a aimsiú, ceachtanna a phleanáil, foclóir a lorg, chomh maith leis an gcruthaitheacht agus an díograis a chóiméad.

Titeann sé orm amháin agus nach bhfuil sórt tacaíocht ar fáil. Ar an bhfoireann féin níl éinne eile á dhéanamh agus ní féidir liom dul chuig éinne. (Cáit)

Tá sé deacair an splanc sin a chóiméad. (Cáit) 
Má tá FCÁT le cur i bhfeidhm i scoileanna lán-Ghaeilge ar bhonn níos leithne, ní mór iniúchadh a dhéanamh ar na féidearthachtaí a bhainfeadh le líonraí tacaíochta a fhorbairt d'fhonn eolas, eispéiris agus áiseanna a roinnt. D'fheadfadh líonraí tacaíochtaí feidhmiú ar bhonn fíorúla nuair a bheadh aithne curtha ag múinteoirí ar a chéile mar atá molta ag Ioannou -Georgiou \& Pavlou (2011). Dá leanfadh na múinteoirí a ghlac páirt sa scéim phíolótach ag forbairt a gcuid saineolais ar FCÁT tríd an tríú teanga, d'fhéadfaidís feidhmiú a mar mheantóirí do mhúinteoirí eile amach anseo.

Ní fhéadfaí ginearálú a dhéanamh ó thuairimí na múinteoirí sa pháipéar seo agus tuairimí múinteoirí eile ar FCÁT mar gheall ar an líon an-bheag múinteoirí a ghlac páirt sa scéim phíolótach agus ar an iliomad comhthéacsanna agus cleachtas a thagann faoin gcontanam den fhoghlaim ábharbhunaithe (Coyle, 2007; Lyster \& Ballinger, 2011; Pérez Cañado, 2018). É sin ráite, tagann tuairimí na múinteoirí sa mhiontionscadal seo le tuairimí múinteoirí eile in Éirinn agus iad ag tosú amach ar FCÁT trí Ghaeilge, trína léiriú go mbaineann dúshlán le cúrsaí pleanála, áiseanna teagaisc, chomh maith le hinniúlacht agus féinmhuinín an mhúinteora féin (Ní Dhiorbháin \& Ní Bhaoill, 2018). Tagann sé chun cinn go láidir sa litríocht gur gá aghaidh a thabhairt ar fhorbairt ghairmiúil do mhúinteoirí agus soláthar saináiseanna teagaisc go mbeidh rath ar an bhfoghlaim i ranganna FCÁT (Ní Chróinín, Ní Mhurchú \& Ó Ceallaigh, 2016; Ní Dhiorbháin \& Ní Bhaoill, 2018).

\subsection{Fís agus Forbairt FCÁT}

Tháinig éagsúlacht na dteangacha Eorpacha a bheadh ar eolas ag múinteoirí bunscoile chun cinn mar bhua agus mar dhúshlán féideartha a bhainfeadh le FCÁT a chur i bhfeidhm go forleathan sna scoileanna lán-Ghaeilge. Mhínigh na múinteoirí go mbeadh teangacha éagsúla ar eolas ag múinteoirí lán-Ghaeilge, agus raon leathan inniúlachta acu sna teangacha sin. Fiú ma tá leibhéal na Ardteiste bainte amach ag múinteoirí ní hionann sin agus a rá go bhfuil an tsainteanga a theastaíonn ó mhúinteoirí FCÁT (Freeman et al., 2015; Morton, 2016, 2017) ar a dtoil acu. Ní fhéadfaí talamh slán a dhéanamh de, ach an oiread go mbeadh eolas ar theanga Eorpach, ag gach múinteoir lán-Ghaeilge. Anuas air sin, is gnáthrud é go mbíonn athruithe foirne sna bunscoileanna lán-Ghaeilge ó bhliain go bliain mar sin bhainfeadh dúshlán le forbairt FCÁT ó bhliain go bliain dá bhfágfaí é faoi scoileanna amháin gan aon mhórpholasaí oideachasúil mar threoir. 
Conas a tharraingítear sin le chéile, nó an é go ndéanann tú é go neamhspleách ó bhliain go bliain, agus seans go bpiocfaidh siad suas rud ó gach múinteoir, atá $i$ gceist? (Príomhoide)

Sa chás go mbíonn éagsúlacht teangacha ag múinteoirí na scoile, d'fhéadfadh clár feasachta teanga a mbeadh spriocanna oideachasúla teanga (Hawkins, 2005) mar aidhm aige, a bheith an-oiriúnach mar chur chuige. Moltar cláir fheasachta teanga do mhúinteoirí nach sainmhúinteoirí teanga iad (Barton, Bragg \& Serratrice, 2009; Jones, Barnes \& Hunt, 2005). Más í an fhís atá ann, ná go mbeadh ábhar scoile amháin á theagasc trí theanga Eorpach ar leith, ó thús go deireadh na bunscoile, cothaíonn inniúlacht na múinteoirí sa sprioctheanga dúshlán maidir le leanúnachas a fhorbairt ó bhliain go bliain. D'eascair smaointí ón bplé go bhféadfadh múinteoir amháin ón scoil uas-sciliú a dhéanamh sa sprioctheanga agus freastal ar ranganna uile na scoile. Léirigh an príomhoide an tuairim go dtaitneodh éagsúlacht poist mar seo le múinteoirí, agus go bhféadfadh múinteoirí sealanna a ghlacadh agus go leathnódh sé go nádúrtha taobh istigh den scoil.

Is dúshlán é an post múinteoireachta agus bíonn tú ag iarraidh an dúshlán a athrú ó am - bíonn siad sa rang 6 nó 7 mbliana teastaíonn athrú agus go minic níl an t-athrú againn le tabhairt dóibh ó am go ham. (Príomhoide)

Is 1 an cheist is mó a eascraíonn ón bplé ná cén múnla FCÁT ab fhearr a d'oirfeadh do shainchomhthéacs na mbunscoileanna lán-Ghaeilge in Éirinn? Ar chóir go mbeadh torthaí foghlama teanga FCAT dírithe ar inniúlacht a chothú in aon sprioctheanga amháin, nó arbh fhearr béim a leagan ar fheasacht a spreagadh ar réimse teangacha? Arbh fhearr díriú ar ábhar scoile amháin nó ar mheascán ábhar? Áitíonn Coyle (2007) gur gá múnlaí FCÁT a fhorbairt a oireann do gach sainchomhthéacs foghlama. Treoróidh na cinntí seo na chéad chéimeanna eile agus an tacaíocht gur ghá a chur ar fáil do mhúinteoirí idir fhorbairt ghairmiúil, líonraí tacaíochta agus saináiseanna. Pé cinntí a ndéanfar, teastóidh mór-pholasaí oideachasúil, pleanáil chórasach, an t-oideachas múinteoirí cuí agus infheistíocht shubstaintiúil. Agus teagasc teangacha Eorpacha sa bhunscoil á phlé, ní mór iniúchadh a dhéanamh ar na féidearthachtaí a bhaineann le teagasc teangacha Eorpacha ar chláir oideachais mhúinteoirí. Agus FCÁT molta mar oideolaíocht le cur le gnóthachtáil foghlaimeoirí sa Ghaeilge ag an mbunleibhéal (Rialtas na hÉireann, 2015, 2019), agus leis na deiseanna foghlama breise a 
bhronnfadh FCÁT tríd an tríú teanga ar fhoghlaimeoirí lán-Ghaeilge, b'fhiú gníomhú i dtreo sainoiliúint ar FCÁT a chur ar fáil thar an gcontanam d'oideachais múinteoirí in Éirinn.

\section{Conclúid}

Ba thionscadal ceannródaíoch a bhí sa taighde seo mar go raibh múinteoirí bunscoile ag cur FCÁT i bhfeidhm tríd an tríu teanga le foghlaimeoirí óga i suíomh an tumoideachais lánGhaeilge. Is taighde ar scála beag a bhí sa taighde agus ní fhéadfaí ginearálú a dhéanamh ó thorthaí an taighde mar go raibh sampla an-bheag múinteoirí agus scoileanna i gceist, agus iad ag tosú amach ar FCÁT don chéad uair riamh. D’fhéadfaí an taighde a uas-scálú amach anseo, ach níos mó múinteoirí a bheith rannpháirteach sa scéim agus breathnóireacht ranga a bheith ina dlúthchuid den mhodheolaíocht taighde. D'fheadfadh an taighde seo a bheith mar bhunús do thaighde níos doimhne, cur i gcás cás staidéir ar mhúinteoirí ar leith nó cas staidéar scoile. In ainneoin theorannacha an taighde seo, tugtar spléachadh dúinn ar chuid de na féidearthachtaí, dúshláin agus saincheisteanna a bheadh le freagairt dá mbeadh FCÁT le cur i bhfeidhm i mbunscoileanna lán-Ghaeilge ar bhonn níos forleithne. Bhronnfadh FCÁT tríd an tríu teanga deis luachmhar ar scoileanna lán-Ghaeilge feidhmiú i dtreo an ilteangachais (An Foras Pátrúnachta, 2018). Thabharfadh FCÁT deis sa bhreis ar fhoghlaimeoirí lán-Ghaeilge feasacht agus suim a chothú i bhfoghlaim teangacha iasachta. Ag eascairt as torthaí an taighde, is léir go bhféadfadh suíomh na scoileanna lán-Ghaeilge a bheith báúil don FCÁT tríd an tríu teanga de bharr taithí na múinteoirí ar an teagasc comhtháite ábhar agus teanga cheana féin. Is údar misnigh iad na múinteoirí lán-Ghaeilge a ghlac páirt sa taighde mar gheall ar an díograis a léirigh siad i leith na foghlama teanga. Tá ábhar machnaimh agus taighde amach romhainn d'fhonn bonn eolais a chruthú do FCÁT i suíomh na mbunscoileanna lán-Ghaeilge.

\section{Tagairtí}

An Foras Pátrúnachta (2018). Ilteangachas: Cur $i$ bhfeidhm CLIL. Ar fáil ag: http://www.foras.ie/wp-content/uploads/2018/06/Ilteangachas.pdf

An Roinn Oideachais \& Scileanna (2020). Statistics primary schools 2019-2020. Ar fáil ag: https://www.education.ie/en/Publications/Statistics/Data-on-Individual-Schools/primary/

An Roinn Oideachais \& Scileanna (2017). Déanann teangacha naisc: Straitéis do theangacha iasachta san chóras oideachais in Éirinn 2017-2026. Ar fáil ag: https://www.education.ie/ga/ScoileannaCol\%C3\%A1ist\%C3\%AD/Eolas/Curaclaim-agus-Siollabas/TeangaIasachta/fls_languages_connect_strategy_ir.pdf 
Ball, D. L., Thames, M. H., \& Phelps, G. C. (2008). Content knowledge for teaching: What makes it special? Journal of Teacher Education, 59(5), 389-407. doi: 10.1177/0022487108324554

Banegas, D. L. (2012). CIIl teacher development: Challenges and experiences. Latin America Journal of Content Language and Integrated Learning, 5 (1), 46-56.

Barton, A., Bragg, J., \& Serratrice, L. (2009). 'Discovering language' in primary school: An evaluation of a language awareness programme. The Language Learning Journal, 37(2), 145-164. doi:10.1080/09571730902928029

Braun, V. \& Clarke, V. (2006) 'Using Thematic Analysis in Psychology'. In: Qualitative Research in Psychology 3 (2): 77-101.

Cohen, L, Manion, L., \& Morrison, K. (2017). Research methods in education (8 ${ }^{\text {th }}$ eag.). Londain and Nua Eabhrac: Routledge.

Comhairle na hEopra (2018). Common European framework of reference for languages: Learning teaching and assessment: Companion Volume with new descriptors. Ar fáil ag: https://rm.coe.int/cefr-companion-volume-with-new-descriptors-2018/1680787989

Comhairle na hEorpa (2008). Próifíl bheartas oideachais teanga: Éire: 2005-2007. Ar fáil ag: https://www.education.ie/ga/Foilseacháin/TuarascálachaOideachais/Próif\%C3\%ADl-Bheartas-Oideachais-Teanga.pdf

Comhairle na hEorpa. (2001). Common European framework of reference for languages: Learning teaching and assessment. Strasbourg: Language Policy Division.

Costa, F., \& D’Angelo, L. (2011). 'CLIL: A suit for all seasons?' Latin American Journal of Content \& Language Integrated Learning 4(1), 1-13. doi:10.5294/laclil.2011.4.1.1 ISSN 20116721

Coyle, D., (2007). Content and language integrated learning: Towards a connected research agenda for CLIL pedagogies. International Journal of Bilingual Education and Bilingualism, 10(5), 543-562.

Coyle, D., Hood, P., Marsh, D. (2010). Content and language integrated learning. Cambridge: Cambridge University Press.

Cummins, J. (2000). Language, Power and Pedagogy: Bilingual children in the crossfire. Clevedon: Multilingual Matters

Dalton-Puffer, C. (2011). Content-and-language integrated learning: From practice to principles? Annual Review of Applied Linguistics, 31, 182.

Dalton-Puffer, C. (2007). Discourse in content and language (CLIL) integrated classrooms. John Benjamins, Amsterdam. 
Darling-Hammond, L. (2000). Teacher quality and student achievement: A review of state policy evidence. Education Policy Analysis Archives, 8 (1), 1-44.

doi:10.14507/epaa.v8n1.2000

Eurydice, (2006). Content and Language Integrated learning CLIL at school in Europe. Ar fáil ag: https://aulaintercultural.org/2006/07/13/content-and-language-integrated-learningclil-at-school-in-europe-2/

Freeman, D., Katz, A., García Gómez, P., \& Burns, A. (2015). English-for-teaching: Rethinking teacher proficiency in the classroom. ELT Journal 69 (2): 129-139

Freeman, D. (1989). Teacher training, development, and decision making: A model of teaching and related strategies for language teacher education. TESOL Quarterly, 23(1), 27-45. doi:10.2307/3587506

Frigols Martin, M. J. (2011). The European framework for CLIL teacher education. Language Teaching, 44(3), 401-402. doi:10.1017/S0261444811000243

Frigols Martín, M.J., Marsh, D., Mehisto, P., \& Wolff, D. (2011). European framework for CLIl teacher education. $\mathrm{Ar}$ fáil ag: https://www.unifg.it/sites/default/files/allegatiparagrafo/20-012014/european_framework_for_clil_teacher_education.pdf

Gilleece, L., Shiel, G., Clerkin,A., \& Millar, D. (2012). Measúnachtaí náisiúnta 2010 ar léitheoireacht Bhéarla agus ar mhatamaitic i scoileanna a bhíonn ag teagasc trí mheán na Gaeilge: Príomhthuarascáil. Baile Átha Cliath. An Foras Taighde ar Oideachas. Harris, J., Forde, P., Nic Fhearaile, S., \& O’ Gorman, M. (2006). An Ghaeilge sna bunscoileanna: Treochtaí náisiúnta fadtéarmacha in inniúlacht/Irish in the primary schools: Long-term national trends in achievement. Baile Átha Cliath: An Roinn Oideachais agus Eolaíochta.

Harris, J. \& Ó Duibhir, P. (2011). Múineadh éifeachtach teanga: Sintéis ar thaighde. Ar fáil ag: https://www.ncca.ie/media/2713/muineadh_eifeachtach_teangacha.pdf

Hawkins, E. (2005). Out of this nettle, drop-out, we pluck this flower, opportunity: Rethinking the school foreign language apprenticeship. The Language Learning Journal, 32(1), 4-17. doi:10.1080/09571730585200141

Ioannou-Georgiou, S., \& Pavlou, P. (2011). (Eag.,). Guidelines for CLIL implementation in primary and pre-primary education. Comhairle na hEorpa: Strasbourg.

Jones, N., Barnes, A., \& Hunt, M. (2005). Thinking through languages: A multi-lingual approach to primary school languages. The Language Learning Journal, 32(1), 63-67. 
Kennedy, M. (1991). A research agenda on teacher learning. East Lansing, MI: National Center for Research on Teacher Learning.

Lamb, P., \& King, G. (2020). Another platform and a changed context: Student experiences of developing spontaneous speaking in French through physical education. European Physical Education Review, 26(2), 515-534. doi:10.1177/1356336X19869733

Lo, Y.Y (2020). Professional development of CLIL Teachers. Singapore: Springer Nature Singapore.

Lyster, R., \& Ballinger, S. (2011). Content-based language teaching: Convergent concerns across divergent contexts. Language Teaching Research, 15(3), 279-288. doi:10.1177/1362168811401150

Mac Dougald, J. (2019). What is next for CLIL professional development? Latin American Journal of Content \& Language Integrated Learning, 12(2), 197-206. doi:10.5294/laclil.2019.12.2.1

Massler, U., Ioannou-Georgiou, S., \& Steiert, C. (2011). Effective CLIL teaching techniques in Ioannou-Georgiou, S., \& Pavlou, P. (2011). (Eag.,). Guidelines for CLIL implementation in primary and pre-primary education. Comhairle na hEorpa: Strasbourg.

Mehisto, P., Marsh, D., \& Frigols, M. J. (2008). Uncovering CLIL: Content and language integrated learning in bilingual and multilingual education. Macmillan, Oxford.

Merino, J. A., \& Lasagabaster, D. (2018). CLIL as a way to multilingualism. International Journal of Bilingual Education and Bilingualism, 21(1), 79-92. doi:10.1080/13670050.2015.1128386

Morton, T. (2017). Reconceptualizing and describing teachers' knowledge of language for content and language integrated learning (CLIL). International Journal of Bilingual Education and Bilingualism, 21(3), 275-286. doi:10.1080/13670050.2017.1383352

Morton, T. (2016). Conceptualizing and investigating teachers' knowledge for integrating content and language in content-based instruction. Journal of Immersion and ContentBased Language Education, 4(2), 144-167. doi:10.1075/jicb.4.2.01mor

Ní Chróinín, D., Ní Mhurchú, S., \& Ó Ceallaigh, T. J. (2016). Off-balance: The integration of physical education content learning and Irish language learning in English-medium primary schools in Ireland. Education 3-13: Contemporary Issues in International Primary Physical Education, 44(5), 566-576. doi:10.1080/03004279.2016.1170404

Ní Dhiorbháin, A. \& Ní Bhaoill, M. (2018). Taithí múinteoirí faoi oiliúint ar an bhfoghlaim chomhtháite ábhar agus teangacha. Comhartaighde (4) 
Ó Ceallaigh, T.J., \& Ní Dhonnabháin, A. (2015). Reawakening the Irish language through the Irish education system: Challenges and priorities. International Electronic Journal of Elementary Education, 8(2), 179-198.

Ó Ceallaigh, T.J., Ó Laoire, M., \& Uí Cheallaigh, M. (2019). Comhtháthú an ábhair agus na teanga san iar-bhunscoil lán-Ghaeilge/Ghaeltachta: I dtreo eispéiris forbartha gairmiúla chun dea-chleachtas a nochtadh: Tuarascáil Taighde ar son na Comhairle um Oideachas Gaeltachta agus Gaelscolaíochta. Baile Átha Cliath: An Chomhairle um Oideachas Gaeltachta \& Gaelscolaíochta.

Ó Duibhir, P. \& Cummins, J. (2012). Towards an integrated language curriculum in early childhood and primary education. Ar fáil ag: https://www.ncca.ie/media/2467/towards an integrated language curriculum in early _childhood_and_primary_education.pdf

Paran, A., (2013). Content and language integrated learning: Panacea or policy borrowing myth? Applied Linguistics Review 4(2). 317-342.

Pérez Cañado, M. L. (2017). Stopping the "pendulum effect" in CLIL research: Finding the balance between Pollyanna and Scrooge. Applied Linguistics Review, 8(1), 79-99. doi:10.1515/applirev-2016-2001

Slyvén, L. K. (2013) 'CLIL in Sweden - Why Does It Not Work? A Metaperspective on CLIL across Contexts in Europe'. In: International Journal of Bilingual Education and Bilingualism 16 (3): 301-320.

Swain, M., \& Johnson, K. R. (1997). Immersion education: International perspectives. Cambridge: Cambridge University Press.

Shulman, L. S. (1987). Knowledge and teaching: Foundations of the new reform. Harvard Educational Review, 57(1), 1-22. doi: 10.17763/haer.57.1.j463w79r56455411

Shulman, L.S. (1986). Those who understand: Knowledge growth in teaching. Educational Researcher. 15(4). 4-14. doi: 10.3102/0013189x015002004 